\title{
Age patterns and sex ratios of adult mortality in countries with high HIV prevalence
}

\author{
Bruno Masquelier ${ }^{a}$, Jeffrey W. Eaton ${ }^{b}$, Patrick Gerland ${ }^{c}$, \\ François Pelletier ${ }^{c}$ and Kennedy K. Mutai ${ }^{d}$
}

\begin{abstract}
Objective: To compare the 2016 United Nations Programme on HIV/AIDS (UNAIDS) modelled estimates of adult mortality in sub-Saharan Africa to empirical estimates.

Design: Age-specific mortality rates were obtained from nationally representative sibling survival data, recent household deaths and vital registration, and directly compared with UNAIDS estimates. Orphanhood prevalence derived from UNAIDS mortality estimates was compared with survey and census reports on the survival of children's parents.
\end{abstract}

Methods: Age-specific mortality rates for adults aged 15-59 years were calculated from Demographic and Health Surveys and deaths reported in censuses or vital registration, adjusted for underreporting, whenever possible. Proportions of orphans were extracted from censuses and surveys for children aged 5-9 years.

Results: UNAIDS estimates were significantly higher than sibling mortality estimates, except among men in countries with very high HIV prevalence. There was a better agreement between rates based on household deaths or vital registration and model outputs. Sex ratios (M/F) of adult mortality were lower in UNAIDS estimates. The modelled orphan prevalence was significantly higher than in surveys and censuses, again with the exception of paternal orphans in countries with very high HIV prevalence. Ratios of paternal-to-maternal orphans were lower in the UNAIDS model than surveys and censuses. Among women, increases in mortality due to AIDS were more concentrated in the age range 25-50 years in model outputs, as compared with empirical estimates.

Conclusion: Discrepancies in levels, sex ratios and age patterns of adult mortality between empirical and UNAIDS estimates call for additional data quality assessments and improvements in estimation methods.

Copyright @ 2017 Wolters Kluwer Health, Inc. All rights reserved.

AIDS 2017, 31 (Suppl 1):S77-S85

Keywords: censuses, demographic and health surveys, life tables, models/ projections, orphans, sub-Saharan Africa

\section{Introduction}

In generalized HIV epidemics in sub-Saharan Africa (SSA), HIV/AIDS has been a major driver of adult mortality trends. Consequently, data on adult mortality could contain valuable information about the timing, scale and age patterns of HIV epidemics in this region. Adult mortality estimates have not been directly incorporated in the process carried out regularly by the United Nations Programme on HIV/AIDS (UNAIDS) to track the demographic impact of the HIV epidemic. This is presumably because these estimates often remain

\footnotetext{
${ }^{a}$ Centre for Demographic Research, Louvain University, Louvain-la-Neuve, Belgium, ${ }^{\mathrm{b}}$ Department of Infectious Disease Epidemiology, Imperial College London, London, United Kingdom, ' United Nations Population Division/DESA, New York, New York, USA, and d National AIDS Control Council, Nairobi, Kenya.

Correspondence to Bruno Masquelier, Centre for Demographic Research, Université Catholique de Louvain (UCL), Place Montesquieu, 1 bte L2.08.03 B-1348-Louvain-la-Neuve, Belgium.

E-mail: bruno.masquelier@uclouvain.be

Received: 3 August 2016; revised: 30 October 2016; accepted: 1 November 2016.
}

ISSN 0269-9370 Copyright $\subset 2017$ The Author(s). Published by Wolters Kluwer Health, Inc. This is an open-access article distributed under the terms of the Creative Commons Attribution-Non Commercial-No Derivatives License 4.0 (CCBY-NC-ND), where it is permissible to download and share the work provided it is properly cited. The work cannot be changed in any way or used commercially without permission from the journal. 
patchy and discrepant when considered at the country level [1]. Civil registration and vital statistics systems often fail to provide full national coverage, and high-quality data on causes of death are not available [2]. Although surveys and censuses provide some direct estimates for adult mortality in SSA, the sparsity of these data and additional uncertainty arising from reporting errors and selection biases mean modelled estimates remain the primary source of information about mortality trends.

At present, AIDS and non-AIDS mortality are estimated in a two-step approach for SSA countries highly affected by HIV. First, non-AIDS life tables are obtained from trends in child mortality, combined with model age patterns that depict the relation between mortality in children and adults expected in the absence of HIV. For the 2016 UNAIDS estimates, these were provided by the United Nations Population Division (UNPD) for 21 countries with high-prevalence levels of HIV. Second, the excess mortality due to AIDS is added on top of these life tables, based on Spectrum, a multistate epidemiological model developed by UNAIDS to estimate key HIV indicators [3] (developed by Avenir Health, Glastonbury, CT, USA). On a country basis, UNPD analysts check the resulting all-cause mortality rates against available empirical data from censuses and surveys. When important discrepancies are apparent with model outputs, the non-AIDS life expectancy is revised and the procedure is repeated until a 'reasonable agreement' is achieved [4]. The resulting estimates are very sensitive to key parameters such as the age patterns and sex ratios of incidence or the model age pattern used to infer the nonAIDS mortality. In addition, as model outputs are checked against empirical estimates based on summary indices of mortality, information contained in the data about age patterns of mortality is not used properly.

The aim of this article is to systematically compare the most recent UNAIDS 2016 estimates of adult mortality and orphanhood with household survey or census measures for 43 countries in SSA (refer to Supplementary Table S1, http://links.lww.com/QAD/B20 for the list of countries). Because of differences in the timing and magnitude of the epidemics, countries are considered in three groups: low-HIV countries include 30 countries where the peak in HIV prevalence among adults aged 15-49 years was lower than $7 \%$, countries with intermediate HIV prevalence include seven countries where the peak in HIV prevalence was between 7 and $17 \%$ and high-HIV countries include six countries, mostly located in southern Africa, where the peak in HIV prevalence was higher than $17 \%$.

Since the onset of the HIV/AIDS epidemic, the number of available data series for adult mortality has greatly increased, thanks to the inclusion of questions on recent household deaths in census and survey questionnaires, and the collection of detailed data on the survival of siblings from women of reproductive age interviewed in Demographic and Health Surveys (DHS). We extracted from these sources the age-specific mortality rates and the probability $45 \mathrm{q} 15$, that is the probability that a person aged 15 years will die before reaching age 60 years if faced with mortality rates prevailing at each age in the period considered. Reports on parental survival also contain useful information on adult mortality. Proportions of respondents whose parents are still alive can be converted into mortality rates based on indirect techniques $[5,6]$. However, HIV has undermined the validity of these techniques, because of the transmission of HIV from mothers to children at birth or through breastfeeding, the lower fertility of seropositive mothers and atypical age patterns of mortality. Adjustments to the orphanhood method have been proposed for use in countries facing HIV/AIDS epidemics, but they concern only maternal orphanhood and are considered provisional $[7,8]$. For this reason, we did not compute indirect mortality estimates from orphanhood but instead directly compared proportions of orphaned children reported in household surveys and censuses with modelled orphanhood prevalence from Spectrum. This programme explicitly accounts for the different sources of AIDS-related biases when computing the number of orphans in childhood based on UNAIDS mortality rates [3,9].

\section{Methods}

\section{Modelled estimates of adult mortality and orphanhood}

Orphanhood and adult mortality rates were extracted from Spectrum files submitted to UNAIDS in 2016. In a few countries, HIV indicators were developed at the subnational level and had to be aggregated to provide national estimates. In 2016, the demographic data for Spectrum were obtained from the 2015 World Population Prospects (WPP) of the UNPD, including non-AIDS life tables for 21 countries.

Historically, life tables estimated by the Population Division for most African countries were largely based on trends in child mortality $(5 \mathrm{q} 0)$ combined with oneparameter model age patterns. Over recent revisions, relational models have been increasingly used to allow for changes in the relationship between mortality in adults and children [10]. When available, various data series are considered, including reports on recent household deaths, orphanhood data, sibling estimates and intercensal survival ratios [11]. In the 2015 WPP, relational models were also used for 20 countries in SSA. In most of these, the HIV prevalence has remained low and there is no need to explicitly model the demographic impact of the epidemic on mortality. In 21 countries with high HIV prevalence, however, two separate processes were modelled: the nonHIV-related mortality (also referred to as the "background mortality') and the mortality due to the HIV/AIDS 
epidemic itself. With few exceptions, the background mortality in countries with high HIV was estimated from one-parameter model age patterns (Supplementary Table S1, http://links.lww.com/QAD/B20). Various model age patterns exist, and the selection of which model to use for a given country is critical, because they can exhibit very different chances of surviving in adulthood for a given level of child mortality (Supplementary Fig. S1, http:// links.lww.com/QAD/B20).

For these 21 countries, the number of AIDS-related deaths was derived from epidemiological modelling and added on top of non-AIDS deaths, based on Spectrum. This programme starts with estimates of HIV prevalence, obtained by fitting a smooth trend through past estimates from surveillance and surveys with the Estimation and Projection Package [12]. From the reconstructed trends in prevalence, the AIM Module of Spectrum calculates incidence rates and AIDS-related deaths, based on estimates and/or assumptions about the age and sex patterns of infection, the patterns of survival after infection, and data on antiretroviral therapy (ART). For countries with lower HIV prevalence, the United Nations Population Division did not produce non-AIDS life tables. In a few cases, such as Burkina Faso and South Sudan, the non-AIDS life expectancy was recalculated by UNAIDS before being entered into Spectrum to obtain a trend that, when combined with Spectrum's estimates of AIDS deaths, would match the all-cause mortality from the WPP 2015 [3]. However, for at least 10 countries, we noted that Spectrum's estimates of AIDS deaths were directly added on top of the all-cause mortality provided by the United Nations Population Division. In Ghana, for example, the life expectancy at birth was estimated at 56.7 years among men by the Population Division for the period 2000-2005, which was similar to Spectrum estimates of the non-AIDS life expectancy, but the addition of AIDS-related deaths reduced the all-cause life expectancy at birth by 2 years. In these countries, adult mortality is likely to be slightly overestimated in UNAIDS 2016 estimates.

Spectrum also produces estimates of the number of orphans among children based on mathematical models [9]. The estimation method starts with the distribution of adult deaths by age and calendar year and consists in estimating how many children were born to those adults and whether these children are still alive and aged less than 18 years at the time of interest. To calculate the number of maternal orphans due to AIDS, the method accounts for the vertical transmission of the virus, the lower fertility of infected mothers and the excess risks of mortality faced by orphans. The HIV status of mothers in the years preceding their death is backcalculated, using a standard pattern of disease progression. The number of maternal orphans due to other causes than AIDS is calculated in a similar way, assuming that their mothers remain uninfected. The estimation of paternal orphans is further complicated by the need to account for the effect on child survival of HIV transmission (from mother to child and between parents) as well as the reduced fertility of partners of infected men [9]. In 2014, the estimation method was revised to account for ART and Prevention of MotherTo-Child Transmission (PMTCT) programmes [3].

\section{Survey and census estimates of adult mortality rates and orphanhood}

Sibling histories were drawn from 91 publicly available DHS datasets in 35 countries (http://www.dhsprogram.com/). These surveys were conducted between 1992 and 2015 (Supplementary Table S2, http://links.lww.com/ $\mathrm{QAD} / \mathrm{B} 20)$. A standardized set of questions was used to elicit an exhaustive list of siblings born to the same mother by birth order. Information was collected about their sex and survival status. Current age was recorded for surviving siblings, whereas age at death and years since death were collected for the deceased. This analysis also included data collected in the men's questionnaires of some DHS.

Age-specific mortality rates were computed directly by dividing the observed number of deaths for each age group and sex by the corresponding person-years of exposure, after weighting the data according to the sample weights. Standard errors were estimated using a Jackknife variance estimation method [13].

Low mortality sibships are overrepresented in sibling histories because the experience of the respondent's siblings is counted multiple times when more than one sibling is interviewed. However, no adjustment for selection biases is needed if one can assume that mortality is not associated with the sibship size and person-years lived by the respondent are included in the calculation [14]. Based on the observation that adult female mortality declines only slightly with the number of sisters surviving in adulthood [15], we did not adjust for selection biases.

Previous analyses of sibling histories have introduced adjustments accounting for the underreporting of deceased siblings in the distant past [16]. These regression-based adjustments have been applied at the regional level and constant across age groups. As there is little decline in completeness of death reporting in the first few years preceding the survey [17], we present here unadjusted estimates for the period referring to $0-5$ completed years before data collection.

We complement DHS estimates with mortality rates calculated from reports on recent household deaths in 45 censuses, 20 large-scale surveys and with vital registration data in Kenya, Zimbabwe and South Africa. Tabulations were obtained from the United Nations Demographic Yearbook or published reports (Supplementary Table S2, http://links.lww.com/QAD/B20). When two censuses 
or large-scale surveys were available for a given country, it was possible to assess the completeness of death reporting based on a suite of demographic approaches known as 'death distribution methods' (DDMs). DDMs compare the age distribution of reported deaths with the age distribution of the population enumerated in the two censuses or surveys to estimate the fraction of deaths that are reported. DDMs rely on strong assumptions that net migration is either negligible or known, that age reporting is accurate and that the completeness of the population enumeration and death reporting are invariant by age. This last assumption is often violated when urban and rural parts of the country have different age structures and varying completeness of death reporting [18]. Three methods are widely used when two inquiries are available: the generalized growth balance (GGB) method [19], the synthetic extinct generations (SEG) method [20] and a hybrid of the two approaches (GGBSEG) [21]. Here we used the hybrid method for 20 censuses in countries where two sets of tabulations were available and the interval between the two data series was not too long (e.g. a decade or less). For South Africa, we mostly used estimates from the Rapid Mortality Surveillance System [22], which are also adjusted for incompleteness of death registration.

Finally, proportions of orphaned children among 5-9year-olds were derived from 60 censuses, 108 DHS, 43 UNICEF's Multiple Indicator Cluster Surveys (MICS) and 19 other nationally representative surveys conducted in SSA. Maternal orphans were defined here as children whose mother is dead, regardless of the survival status of their father. Paternal orphans were defined in a similar way. Missing or unknown responses were discarded, under the assumption that ignorance of orphanhood status is not associated with the risk of being orphan [23]. Orphanhood estimates are biased by the 'adoption effect', which refers to the fact that some fostered orphans are misclassified as nonorphans. In the presence of adults, interviewers may not probe whether they are the biological parents of children observed in the household [24]. Foster parents may also inadvertently claim adopted orphans as their own offspring. The usage of terms pertaining to biological parents to refer to larger circles of kins and the practice of child fostering can add to the confusion.

\section{Statistical analysis}

We computed ratios of modelled estimates to survey or census-based estimates of orphanhood and adult mortality for each data series and report here the median value of these ratios. We used Wilcoxon signed-rank tests for paired data to test for significant differences between types of estimates [25]. We also used linear mixed-effects models to identify whether differences between both types of estimates vary by sex, group of countries and with HIV incidence.

\section{Results}

\section{Levels and sex ratios of adult mortality}

Figure 1a compares the probability $45 \mathrm{q} 15$ as estimated from sibling survival histories with UNAIDS 2016 estimates for each sex. Supplementary Figs. S2, http:// links.lww.com/QAD/B20 to S4, http://links.lww.com/ QAD/B20 present some country-specific plots. The agreement between both series of estimates clearly varies by sex and across groups of countries. Table 1 displays the median ratios of mortality rates between modelled estimates and estimates from surveys, censuses and vital registration (columns 1 and 2). The median sex ratios in both sets of estimates are presented in columns 3 and 4 . In countries with relatively low HIV prevalence, the median ratios were 1.28 for women and 1.14 for men based on sibling histories (Wilcoxon signed-rank tests, $P<0.001$ for both sexes), suggesting that adult mortality is lower than assumed in models, especially among women (Table 1). The same is true in countries with intermediate HIV prevalence (mostly located in eastern Africa), with a median ratio of 1.2 for women $(P<0.001)$ and 1.12 for men $(P=0.03)$. By contrast, in countries with very high HIV prevalence in southern Africa (and Zimbabwe), the median ratio was 1.16 for women $(P=0.01)$ and modelled mortality estimates were lower than sibling histories among men (0.91), although the difference was NS $(P=0.10)$. In the three groups of countries, sex differentials in adult mortality were significantly larger than in UNAIDS 2016 estimates $(P<0.001)$. In countryspecific plots for Zambia, Namibia and Zimbabwe (Supplementary Figs. S3, http://links.lww.com/QAD/ B20 and S4, http://links.lww.com/QAD/B20), there was no distinct reduction of the women advantage in adult mortality as the share of AIDS-related deaths increased. Yet, in UNAIDS estimates, differences in sexspecific 45q15 have been narrowing, with even some excess women mortality for some time.

Figure $1 \mathrm{~b}$ displays a comparison of the probability $45 \mathrm{q} 15$ extracted from reports on recent household deaths or vital registration and UNAIDS estimates. Mortality rates matched fairly well in countries with low and intermediate HIV prevalence. In countries with very high HIV prevalence, UNAIDS estimates provided higher female mortality $(P=0.02)$. UNAIDS estimates for men were lower than surveys, censuses and vital registration, except in South Africa. There were also significant differences in sex ratios $(\mathrm{M} / \mathrm{F})$ of adult mortality in these countries with very high HIV prevalence.

Finally, the prevalence of orphanhood among 5-9-yearolds observed in surveys and censuses is compared with Spectrum estimates in Fig. 1c. Proportions of maternal and paternal orphans obtained from Spectrum were significantly higher than those derived from surveys and censuses in countries with low $(P<0.001)$ and intermediate HIV prevalence $(P<0.001$ for mothers and $P=0.05$ for 
(a)

45q15 from UNAIDS 2016 and DHS sibling histories
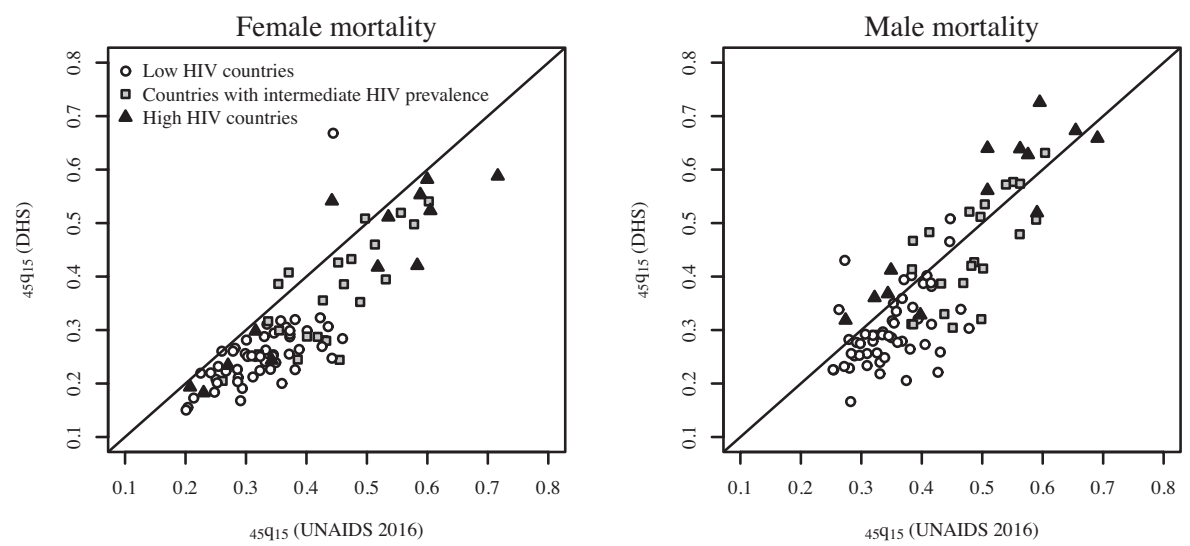

(b)

45q15 from UNAIDS 2016 and recent household deaths or VR
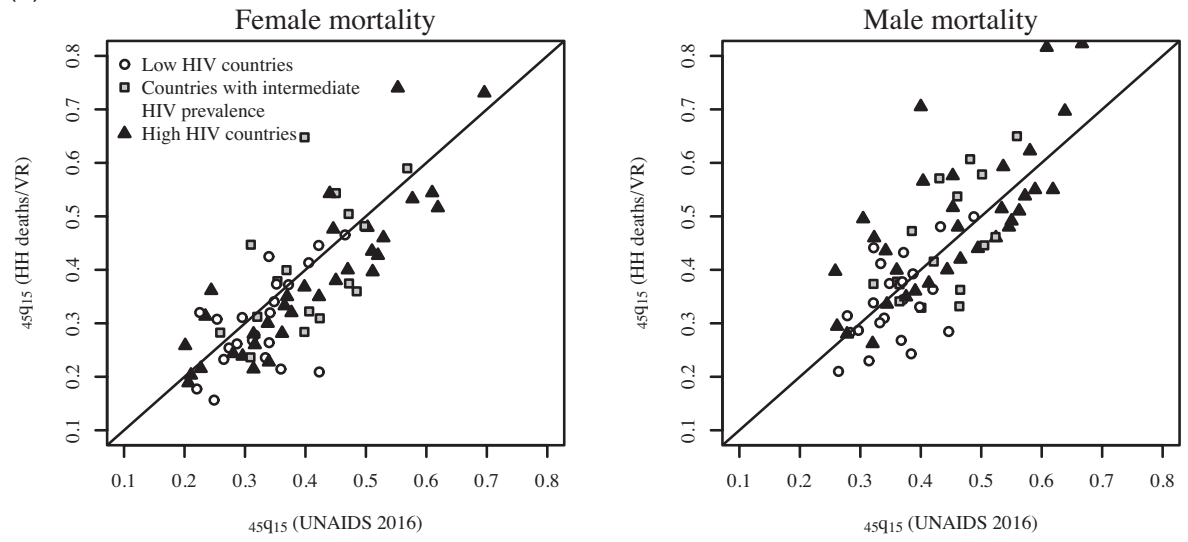

(c)

Orphanhood prevalence from UNAIDS 2016 and surveys or censuses
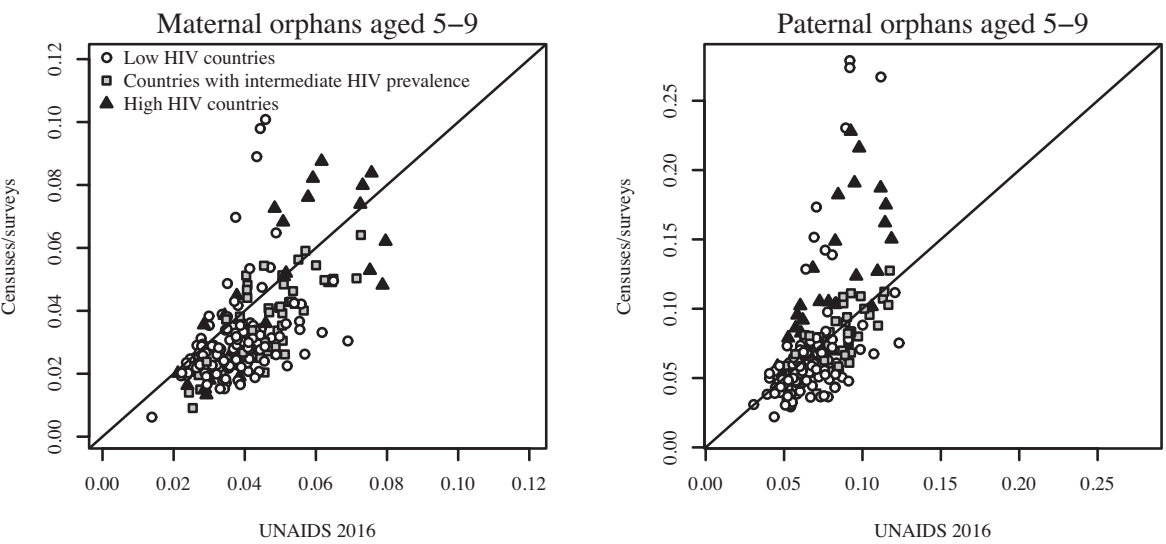

Fig. 1. Comparison of empirical and model-based estimates of adult mortality and orphanhood. (a) Estimates of the probability 45q15 from sibling survival histories and the United Nations Programme on HIV/AIDS 2016; (b) estimates of the probability $45 q 15$ from recent household deaths or vital registration and the United Nations Programme on HIV/AIDS 2016; (c) prevalence of orphanhood among 5-9-year-olds observed in surveys and censuses and the United Nations Programme on HIV/AIDS.

fathers). Similarly to what was observed with adult mortality, median ratios were lower for paternal orphans. In countries where HIV peaked at $17 \%$ or more, the modelled prevalence was significantly lower than from survey or census for paternal orphanhood $(P<0.001)$. Ratios of maternal-to-paternal orphanhood compare different age groups of parents, as fathers are older at the birth of their children because of differences in ages at marriage. It is therefore expected to observe larger sex ratios in orphanhood prevalence than in adult mortality rates in both empirical and model-based estimates, as can be seen in Table 1. However, differences between paternal 
Table 1. Median ratios between modelled estimates and survey or census-based estimates of adult mortality and orphanhood (columns 1 and 2) and median sex ratios (columns $3-5$ ).

\begin{tabular}{|c|c|c|c|c|c|c|}
\hline \multirow[b]{2}{*}{ Data source } & \multirow[b]{2}{*}{ Group } & \multicolumn{2}{|c|}{$\begin{array}{l}\text { Median ratios of } \\
\text { modelled to empiri- } \\
\text { cal estimates }\end{array}$} & \multicolumn{3}{|c|}{ Sex ratios (M/F) } \\
\hline & & $\begin{array}{l}\text { Women } \\
\text { (1) }\end{array}$ & $\begin{array}{l}\text { Men } \\
(2)\end{array}$ & $\begin{array}{l}\text { UNAIDS } \\
\text { (3) }\end{array}$ & $\begin{array}{l}\text { Surveys, censuses and } \\
\text { vital registration } \\
\text { (4) }\end{array}$ & $\begin{array}{c}\text { Sign. } \\
(5)\end{array}$ \\
\hline \multirow[t]{3}{*}{ Sibling survival data } & (1) Low HIV & $1.28^{\mathrm{a}}$ & $1.14^{\mathrm{a}}$ & 1.07 & 1.24 & $\mathrm{a}$ \\
\hline & (2) Intermediate HIV & $1.2^{\mathrm{a}}$ & $1.12^{b}$ & 1.08 & 1.22 & a \\
\hline & (3) High HIV & $1.16^{\mathrm{b}}$ & 0.91 & 1.09 & 1.31 & a \\
\hline \multirow{3}{*}{$\begin{array}{l}\text { Recent household deaths } \\
\text { and vital registration }\end{array}$} & (1) Low HIV & 1.07 & 1.02 & 1.09 & 1.12 & \\
\hline & (2) Intermediate HIV & 0.99 & 0.97 & 1.09 & 1.18 & \\
\hline & (3) High HIV & $1.12^{\mathrm{b}}$ & 0.99 & 1.15 & 1.33 & a \\
\hline \multirow{3}{*}{ Orphanhood } & (1) Low HIV & $1.31^{\mathrm{a}}$ & $1.13^{\mathrm{a}}$ & 1.78 & 2.02 & a \\
\hline & (2) Intermediate HIV & $1.28^{\mathrm{a}}$ & $1.07^{\mathrm{C}}$ & 1.83 & 2.17 & a \\
\hline & (3) High HIV & 1.11 & $0.72^{\mathrm{a}}$ & 1.72 & 2.59 & $\mathrm{a}$ \\
\hline
\end{tabular}

Note: Significance levels in columns 1 and 2 were obtained from Wilcoxon signed-rank tests of the null hypothesis that the distribution of differences between (rank-transformed) model, and survey estimates are symmetric around 0 . Significance levels in column 5 refer to differences between model and survey-based/census-based sex ratios $\left(0-0.001^{\mathrm{a}} ; 0.01-0.05^{\mathrm{b}} ; 0.05-0.1^{\mathrm{c}}\right)$.

and maternal orphan rates were much larger in surveys and censuses than in model-based estimates.

According to regression analysis presented in Appendix F, http://links.lww.com/QAD/B20, discrepancies between model and empirical estimates point to systematic biases in survey or census data (irrespective of the HIV epidemic), but also to problems with the modelling of AIDS-related mortality (with a possible too large femaleto-male sex ratio of HIV incidence) and/or with an underestimated non-AIDS mortality among men in countries with very high HIV prevalence.

\section{Age patterns of adult mortality}

Figure 2 displays ratios of modelled to DHS estimates of adult mortality broken down by sex, group of country, age group and level of HIV prevalence (refer to Supplementary Fig. S6, http://links.lww.com/QAD/B20 for similar boxplots based on household deaths and vital registration). Disregarding the differences in the overall level, age patterns of mortality are fairly comparable in countries with low HIV prevalence, as the ratios deviate from parity by a similar extent across age groups. In countries with intermediate HIV prevalence, the peak in women death rates between ages 25 and 50 years is slightly more protruding in model outputs data than in DHS data. When HIV prevalence reaches $10 \%$, these differences are accentuated, and DHS even tend to provide higher mortality rates among women aged less than 25 years. Among men, ratios broken down by age do not show a clear pattern, except above age 50 years in which DHS tend to provide higher mortality rates when HIV prevalence increases. Mortality rates available from sibling histories in countries with very high HIV prevalence are already dominated by AIDS mortality, because few countries have conducted DHS in the 1990s. Again, mortality rates seem to increase less rapidly with age in DHS than in modelled outputs among women. There is a better agreement when looking at age patterns among men, notwithstanding the overall differences in $45 q 15$.

\section{Discussion}

The current article compared UNAIDS 2016 estimates of orphanhood and adult mortality with data from surveys, censuses and vital registration in SSA. We observed discrepancies in levels, sex ratios and age patterns of adult mortality.

Some of these deviations are indicative of systematic biases in empirical measurements. Even when restricting the analysis of sibling histories to the first 5 completed years before data collection, estimates could be affected by reporting errors such as omissions of siblings, and age or date errors [26]. Record linkage studies with prospective mortality data collected in Senegal suggest that overall, sibling histories could lead to a $20 \%$ underestimate of the probability 45q15 [27]. Observed proportions of orphans are also known to underestimate the true orphan prevalence, primarily because of the 'adoption effect'. This effect is deemed more pronounced for maternal orphans [28]. This is because paternal orphans are more likely to live with their surviving mother, and widowers are more likely to remarry. Thus, part of the differences in sex ratios of parental survival can be ascribed to more pervasive misreports when information is elicited about mothers. The high rates of paternal orphanhood could also be due to misreporting of absent fathers as presumed dead. However, sex ratios of mortality are also larger in sibling survival histories than in model-based estimates, and there is no evidence that survey reports on the survival of sisters is less reliable than on survival of brothers [17]. 

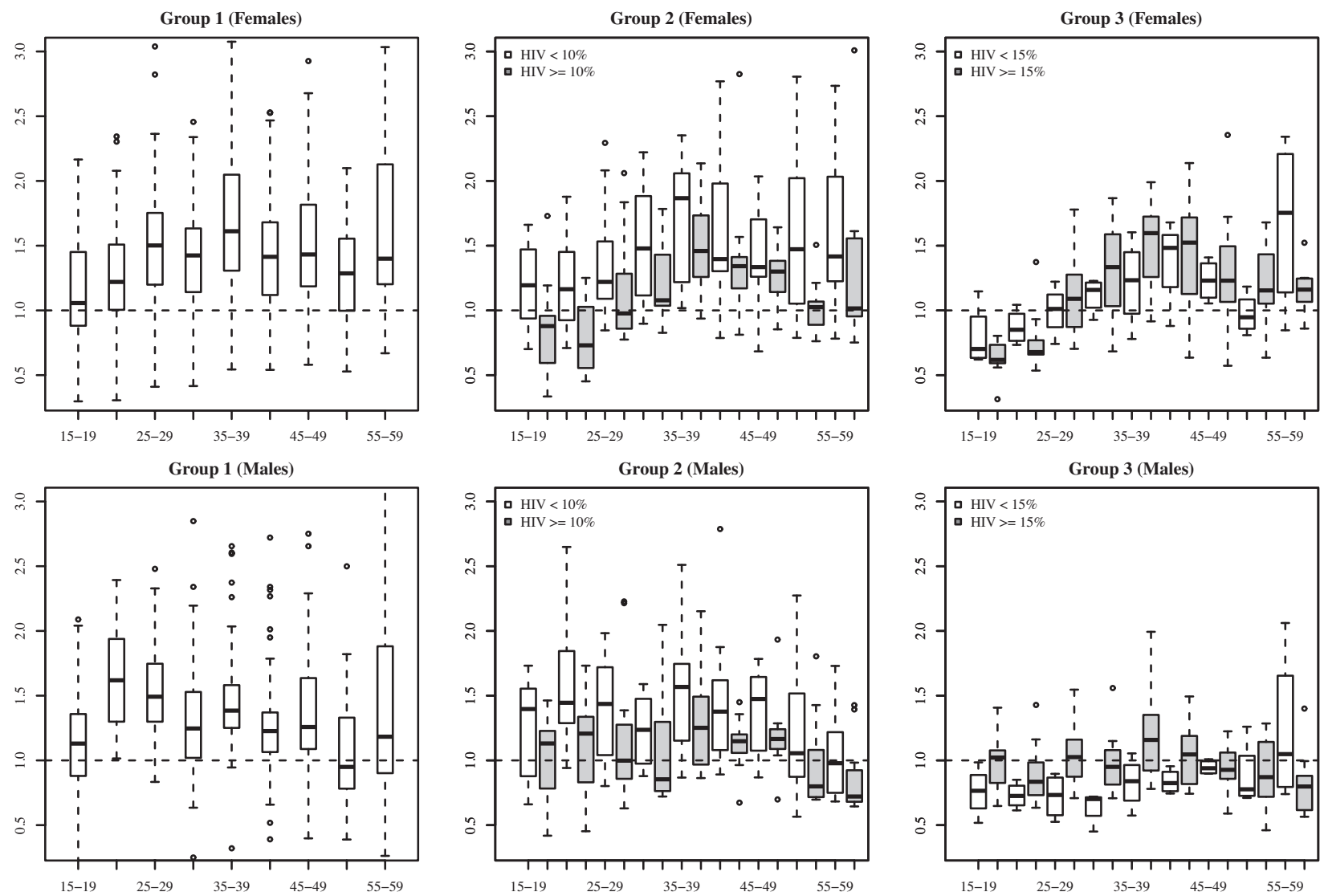

Fig. 2. Ratios of the United Nations Programme on HIV/AIDS 2016 age-specific mortality rates to Demographic and Health Surveys estimates derived from sibling histories, by sex, group of country and level of HIV prevalence.

Some deviations between empirical and model-based estimates may point to errors in the female-to-male sex ratios of HIV incidence or the non-AIDS mortality. In particular, in southern Africa, both sibling survival and paternal orphanhood suggest that male mortality is higher than represented in model estimates. It has become increasingly problematic to infer non-AIDS mortality rates by matching indices of child survival onto standard mortality schedules, because the resulting estimates are very sensitive to the selection of an age pattern of mortality, model life tables currently in use are primarily drawn from the historical experience of Western countries and mortality trends observed in child mortality may not reflect trends in mortality of the uninfected adult population (in particular as substantial efforts have been devoted to reducing child mortality through specific interventions in recent decades).

Finally, this analysis also suggests discrepancies arising from the modelling of the demographic impact of HIV. According to surveys, censuses and vital registration, the bulge in age patterns of mortality due to AIDS is less concentrated than what is depicted by models for women. This results in higher mortality rates among women aged 15-24 years in empirical estimates when HIV reaches
$10 \%$, as compared to model estimates. In addition, model estimates often show a peak in female mortality in the age group 35-39 years when HIV passes this threshold, whereas mortality rates remain the highest at age 50 years and above in empirical estimates. These differences in age patterns cannot be attributed to misstatements of ages at death, because they should then be apparent in countries with low HIV prevalence too. During the course of the HIV epidemic, the 'AIDS hump' of mortality is expected to broaden and gradually shift towards older ages [29]. This shift is mainly driven by a reduction of mortality following the increasing availability of antiretroviral treatments, and the aging of the infected population, which are both taken into account in models. But even before ART, such changes can also be supported by modifications in the age profile of HIV incidence, and deviations from empirical measurements could point to inaccurate assumptions about modifications in the age profile of new infections (or changes in sex ratios of HIV incidence) as the epidemic matures.

Our analysis is similar to an earlier comparison of household survey data with 2001 UNAIDS estimates of orphanhood [25]. Proportions of maternal orphans in MICS and DHS were consistently lower than model predictions (by $40 \%$ on 
average), irrespective of the HIV prevalence. Paternal orphan prevalence was more in agreement with model outputs. At that time, the authors ascribed these discrepancies to a combination of overestimation of background adult mortality by the United Nations and underreporting of orphanhood status because of the adoption effect. They concluded that reducing adult mortality from causes other than AIDS would produce a closer agreement with survey data. Our conclusions differ. Post-hoc adjustments to either the AIDS or nonAIDS component of adult mortality are unlikely to reduce differences with survey or census data in a consistent way. Deviations from model outputs vary by sex, by region and according to the data source, in such a way that it is very difficult to understand what caused the differences in estimates. Rather than adjusting the AIDS and non-AIDS mortality somewhat independently, future revisions of estimates should aim to directly model age-specific mortality rates from all causes combined. For example, the model used by the United Nations Inter-agency Group for Child Mortality Estimation to synthesize various estimates of under-five mortality allows for reporting biases in data series based on a multilevel model $[30,31]$. Specific data series are allowed to be entirely biased upwards or downwards, and recall biases in birth histories are modelled as a linear function of the time elapsed between each retrospective reference period and the survey. Such statistical models, informed by data quality assessments of surveys and censuses, could be expanded to adult mortality. Improving adult mortality estimation could yield insights into key parameters of the dynamic of the HIV epidemic, such as changes in age and sex patterns of incidence over time and changes in ART coverage.

\section{Acknowledgements}

B.M. analysed the data from surveys and censuses and drafted the manuscript. J.W.E. analysed UNAIDS estimates. P.G. developed the database of adult mortality measurements. F.P. and K.K.M. critically reviewed the manuscript. The authors wish to thank Mary Mahy for providing the most recent UNAIDS estimates. The views expressed in this article are those of the authors and are not necessarily those of the United Nations.

\section{Conflicts of interest}

There are no conflicts of interest.

\section{References}

1. Reniers G, Masquelier B, Gerland P. Adult mortality in Africa. In: Rogers R, Crimmins E, editors. International handbook of adult mortality. New York: Springer; 2011.

2. Mikkelsen L, Phillips DE, AbouZahr C, Setel PW, de Savigny D, Lozano $\mathrm{R}$, Lopez AD. A global assessment of civil registration and vital statistics systems: monitoring data quality and progress. Lancet 2015; 386:1395-1406.
3. Stover J, Andreev K, Slaymaker E, Gopalappa C, Sabin K, Velasquez $C$, et al. Updates to the spectrum model to estimate key HIV indicators for adults and children. AIDS 2014; 28 (Suppl 4):S427-S434.

4. United Nations. World population prospects: the 2004 revision, vol. III: analytical report. New York: Population Division, Dept. of International Economic and Social Affairs, United Nations; 2005.

5. Brass W, Hill K. 'Estimating adult mortality from orphanhood,' in Proceedings of the International Population Conference: Liège (I. U. for the Scientific Study of Population, ed.), vol. 3, pp. $111-123,1973$.

6. Blacker J. The estimation of adult mortality in Africa from data on orphanhood. Popul Stud 1977; 31:107-128.

7. Timæus I, Nunn A. Measurement of adult mortality in populations affected by AIDS: an assessment of the orphanhood method. Health Transit Rev 1997; 7:23-43.

8. Timæus I. Indirect estimation of adult mortality from orphanhood. In: Moultrie T, Dorrington R, Hill A, Hill K, Timæus I, Zaba B,editors. Tools for demographic estimation Paris: International Union for the Scientific Study of Population; 2013.

9. Grassly N, Timæus I. Methods to estimate the number of orphans as a result of AIDS and other causes in subSaharan Africa. I Acquir Immune Defic Syndr 2005; 39:635675.

10. United Nations. 'World population prospects: The 2012 revision, methodology of the United Nations population estimates and projections'. Working paper no. ESA/P/ WP.235.2014.

11. Hill $K$, Choi $Y$, Timæus I. Unconventional approaches to mortality estimation. Demogr Res 2005; 13:281-300.

12. Brown T, Bao L, Eaton JW, Hogan DR, Mahy M, Marsh K et al. Improvements in prevalence trend fitting and incidence estimation in EPP 2013. AIDS 2014; 28 (Suppl 4):S415S425.

13. Pedersen J, Liu J. Child mortality estimation: appropriate time periods for child mortality estimates from full birth histories. PLoS Med 2012; 9:e1001289.

14. Trussell J, Rodriguez G. A note on the sisterhood estimator of maternal mortality. Stud Fam Plann 1990; 21:344-346.

15. Masquelier B. Adult mortality from sibling survival data: a reappraisal of selection biases. Demography 2013; 50: 207-228

16. Obermeyer Z, Rajaratnam J, Park C, Gakidou E, Hogan M, Lopez A, Murray C. Measuring adult mortality using sibling survival: a new analytical method and new results for $\mathbf{4 4}$ countries, 1974-2006. PLoS Med 2010; 7:e1000260.

17. Masquelier B, Reniers G, Pison G. Divergences in mortality trends in sub-Saharan Africa: survey evidence on the survival of children and siblings. Popul Stud 2014; 68:161177.

18. Dorrington $\mathrm{R}$. The generalized growth balance method. In: Moultrie T, Dorrington R, Hill A, Hill K, Timæus I, Zaba $\mathrm{B}$,editors. Tools for demographic estimation Paris: International Union for the Scientific Study of Population; 2013.

19. Hill K. Estimating census and death registration completeness. Asian Pac Popul Forum 1987; 1:8-13.

20. Bennett N, Horiuchi S. Mortality estimation from registered deaths in less developed countries. Demography 1984; 21:217-234.

21. Hill K, You D, Choi Y. Death distribution methods for estimating adult mortality: sensitivity analysis with simulated data error. Demogr Res 2009; 21:235-254.

22. Dorrington R, Bradshaw D, Laubscher R, Nannan N. Rapid mortality surveillance report 2014. Cape Town: South African Medical Research Council; 2015.

23. Beegle K, Filmer D, Stokes A, Tiererova L. Orphanhood and the living arrangements of children in sub-Saharan Africa. World Dev 2010; 38:1727-1746.

24. Blacker J. Experience in the use of special mortality questions in multipurpose surveys: the single-round approach. In: United Nations. editor. Data bases for mortality measurement New York: United Nations; 1984. pp. 79-89.

25. Grassly N, Lewis J, Mahy M, Walker N, Timæus I. Comparison of household-survey estimates with projections of mortality and orphan numbers in sub-Saharan Africa in the era of HIV/ AIDS. Popul Stud 2004; 58:207-217. 
26. Helleringer S, Pison G, Masquelier B, Kanté A, Douillot L, Duthé $\mathrm{G}$, et al. Improving the quality of adult mortality data collected in demographic surveys: validation study of a new siblings' survival questionnaire in Niakhar, Senegal. PLOS Med 2014; 11:e1001652.

27. Helleringer S, Pison G, Kanté M, Duthé G, Andro A. Reporting errors in survey data on adult mortality: results from a record linkage study in Senegal. Demography 2014; 51:387-411.

28. Robertson L, Gregson S, Madanhire C, Walker N, Mushati $P$, Garnett G, Nyamukapa C. Discrepancies between UN models and DHS survey estimates of maternal orphan prevalence: insights from analyses of survey data from Zimbabwe. Sex Transm Infect 2008; 84 (S(1)):57-62.
29. Sharrow DJ, Clark SJ, Collinson MA, Kahn K, Tollman SM. The age pattern of increases in mortality affected by HIV: Bayesian fit of the Heligman-Pollard model to data from the Agincourt HDSS field site in rural northeast South Africa. Demogr Res 2013; 29:1039-1096.

30. Alkema L, New JR, Pedersen J, You D, You D, Bastian P, et al. Child mortality estimation 2013: an overview of updates in estimation methods by the United Nations Interagency Group for Child Mortality Estimation. PLOS One 2014; 9:e101112.

31. Alkema L, New JR. Global estimation of child mortality using a Bayesian b-spline bias-reduction model. Ann Appl Stat 2014; 8:2122-2149. 